\title{
THE CONSERVATIVE SURGICAL TREATMENT OF IDIOPATHIC ASEPTIC NECROSIS OF THE FEMORAL HEAD
}

\author{
M. Kerboul, J. Thomine, M. Postel and R. Merle d’Aubigné, Paris, France \\ From Hôpital Cochin, Paris
}

Most of the patients struck by idiopathic necrosis of the femoral head are men between thirty and sixty years of age in an active period of life. Although about 25 per cent improve spontaneously after one or two years and have a tolerable disability, most of them worsen; in half the cases the other hip becomes involved during the first or second year. Some degree of collapse of the head occurs in 90 per cent, leading eventually to a progressive arthrosis.

Medical treatment is ineffectual. The use of crutches, and even of bed rest with or without traction, does not prevent collapse of the head. Drilling of the trochanter and neck as advised by Ficat and Arlet (1972) relieves the pain if performed at an early stage. The frequent finding of a very high intramedullary venous pressure by those authors provides a rational basis for this treatment, but it has not been used at Hôpital Cochin.

In 1963 Merle d'Aubigné, Mazabraud and Cahen and in 1965 Merle d'Aubigné, Postel, Mazabraud, Massias and Gueguen reported 139 operations in this Journal, including the results of thirteen arthrodeses, thirty-four femoral head replacements, ten cup arthroplasties and sixty osteotomies. The present paper gives the results of surgical treatment in 240 hips.

Replacement of the necrotic head alone by a metallic prosthesis had been used up to 1968 in eighty-four hips (Aubriot 1973). Forty-six have now been reviewed three to ten years after the operation. The functional results are classified as excellent in only three cases, good in sixteen, fair in twelve (not because of pain but because of stiffness or limited walking), and poor in fifteen. Two prostheses were removed for sepsis. Up to date five have had total replacement on account of pain.

Many of those failures appeared to result from deterioration of the acetabulum. Such change was evident radiologically in all cases where the joint space was absent before operation and in half of the others, including hips where the joint space was normal or even wider than normal. Although some hips have a reasonably good result in spite of displacement of the prosthesis into the pelvic bone, the clinical and radiological evolution strongly suggests a preference for total prosthesis. But total replacement is also an adventure, especially under the age of forty, as about half the patients are.

This is why persistent attempts have been made in our department, when the patient's disability demanded active measures, to use operations that preserve the femoral head in the hope of limiting the collapse and encouraging incorporation of the necrotic part. At the present time, apart from drilling, of which we have no experience, and curettage plus grafting, used in only five cases, two operations appear to offer that hope: intertrochanteric osteotomy and "adjusted cup" arthroplasty, an operation used in more than 100 cases since 1964.

The aim of this paper is to present the results of these two operations as found in a review of two consecutive series of cases-ninety-two osteotomies followed for one to thirteen years and eighty "adjusted cup" arthroplasties followed from one to six years.

\section{OSTEOTOMY}

Seventy-six patients (sixty-seven men, nine women) aged between twenty-three and sixtythree (average forty-four), with 112 affected hips, underwent ninety-two osteotomies (Kerboull 1973). Although most cases were operated on early (68 per cent in the first year), some collapse of the femoral head was already present in 87 per cent.

Evaluation of the extent of the necrosis was attempted by measuring the arc of the

VOL. 56 B, NO. 2, MAY 1974 
surface involved by necrosis in antero-posterior and lateral radiographs of the head, and adding the two angles (Fig. 1). The extent was considered large when the sum was 200 degrees or more and small when 160 degrees or less. The figures so obtained were: large, 47 per cent: medium, 38 per cent; and small, 15 per cent.

Osteotomy was performed in forty unilateral and thirty-six bilateral cases. Of the latter, fifteen had osteotomy on both sides, eight had osteotomy on one side only and no operation

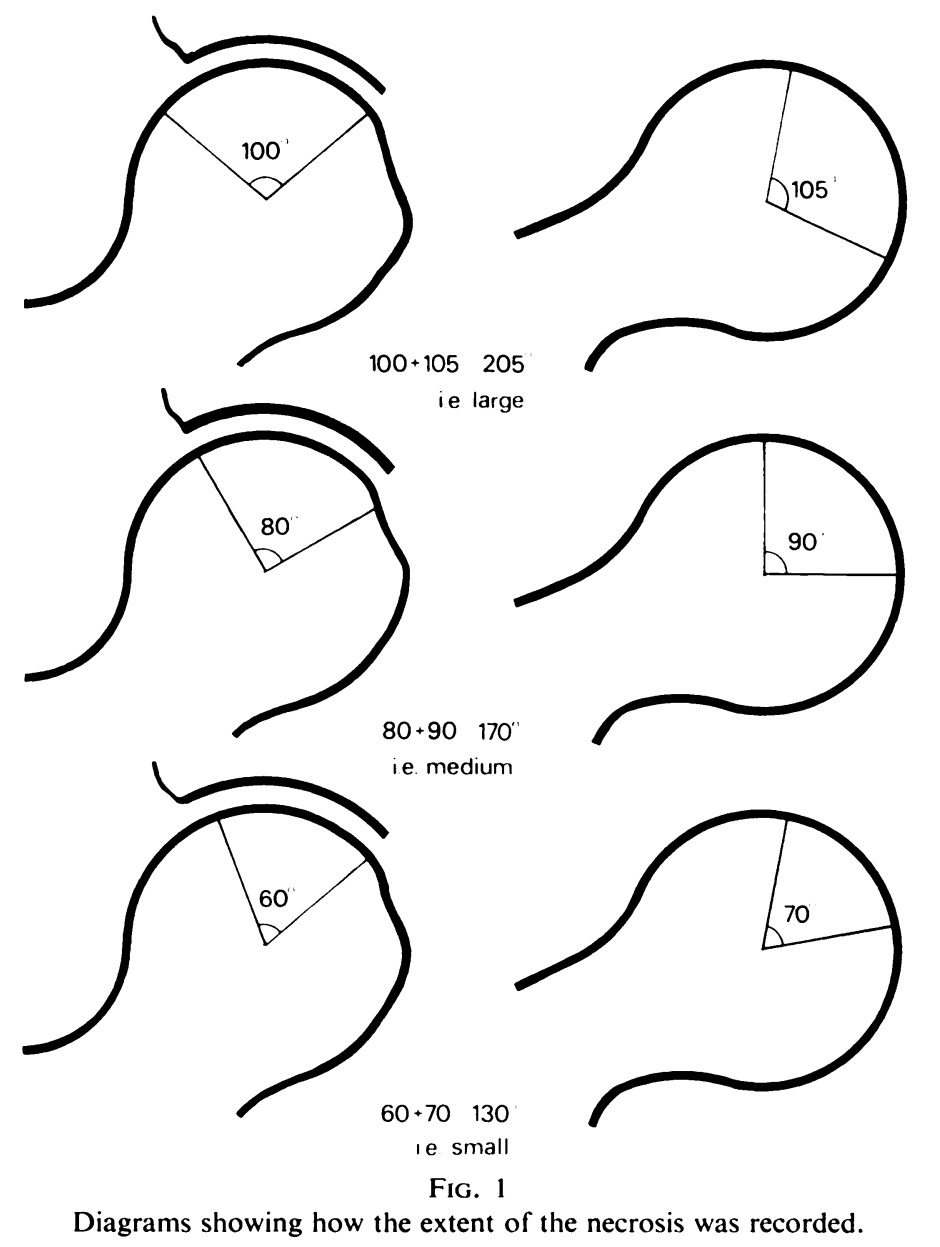

on the other hip, nine had osteotomy on one side and an arthroplasty on the other, three had osteotomy and an arthrodesis, and one had an osteotomy and a tenomyotomy.

The aim of the osteotomy was to remove the necrotic part of the head from the zone of maximum pressure and replace it by the sound postero-lateral part. In order to achieve this after oblique or transverse intertrochanteric osteotomy, the shaft of the femur was brought into adduction and flexion, which means a shift of the head and neck into abduction and extension, so bringing the necrotic part medially and inferiorly (Merle d'Aubigné and Frain 1972). For an oblique osteotomy the bone fixation was by bolts, for a transverse osteotomy by blade plate. Active movement was started early but weight-bearing was delayed for three to four months.

\section{RESULTS OF OSTEOTOMY}

After one year, pain was completely relieved or nearly so in 89 per cent of the cases. Movement was always preserved, but the ability to walk was still more or less impaired in 40 per cent. 
After five years only thirty-nine patients with forty-seven osteotomies could be reviewed: ten had died and twenty-seven were lost sight of. The proportion of painless hips was 59 per cent at five years, and only 47 per cent at six years. Function deteriorated similarly, the proportion of good functional results falling from 47 per cent at five years to 32 per cent at six years. The radiographs showed that in 33 per cent the necrotic part appeared revitalised and in 25 per cent unchanged; further collapse occurred in 42 per cent. Secondary arthrosis was present in 90 per cent. Altogether 40 per cent of the cases could be considered as failures, either immediate (15 per cent), early ( 7 per cent), or late (18 per cent). Nevertheless, 60 per cent of the patients were practically free from pain five years after the osteotomy.

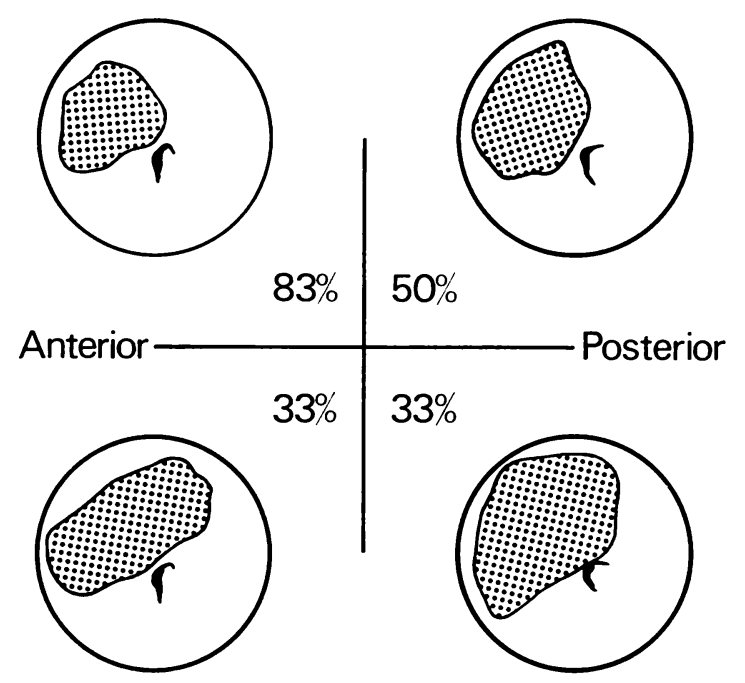

Fig. 2

A diagram showing the influence of size and situation of the necrosis on the results of osteotomy.

The question is the relationship between success and failure on the one hand, and on the other, the actual achievement of the mechanical aim of the operation-relief of pressure on the necrotic part of the head. For success two conditions appear to be necessary: firstly, a change of position of the femoral head by the desired amount; and secondly, a suitable size and situation of the area of necrosis. The angulation after osteotomy was exactly as planned in 45 per cent of the operations but only approximately so in the remainder. The size of the necrotic part has a definite but limited influence on the result, its situation being much more important (Fig. 2). When the necrosis extends posteriorly, only one case out of three obtains a good result. When the sequestration is situated on the anterior slope of the head with the posterior surface intact, the proportion of good results rises to one out of two if there is a small sound supero-lateral surface, and to four out of five if there is a large sound surface supero-laterally as well as posteriorly.

It was attempted to judge from the radiographs to what extent the necrotic zone had been relieved from maximum pressure, either completely, partially or not at all. The findings are as follows. Pain was completely or nearly completely relieved in all cases where the sequestrum was completely removed from the zone of pressure, in 70 per cent when partially removed, and in only 46 per cent when not removed.

Comment-Success can be expected from intertrochanteric osteotomy in cases of idiopathic necrosis with a moderate tendency to advance, provided the radiographs show that a limited portion of the articular surface of the head is necrotic. The antero-posterior view should demonstrate an arc of at least 20 degrees of sound surface lateral and superior to the necrotic 
part; in abduction this part should be covered by acetabulum. In the lateral view, the necrotic portion should not involve the posterior aspect. According to Rydell (1966), the zone of high pressure is situated over the superior part of the anterior wall of the acetabulum. The displacement after osteotomy therefore must bring the postero-superior part of the head forwards and the lateral part inwards. This can be achieved if the femoral shaft is fixed to the upper fragment in a position of adduction and flexion.

In our series about half of the femoral heads were involved too extensively to give a reasonable chance of success. Consequently another surgical means of protecting the necrotic femoral head has been attempted, namely the "adjusted cup" arthroplasty.

\section{“ADJUSTED CUP” ARTHROPLASTY}

Cup arthroplasty following Smith-Petersen's principle of a cup loose both on the head and in the acetabulum was used in a few cases with poor results. The rationale of the "adjusted cup" is quite different. The aim is to protect the necrotic area by a cup fitting snugly on the

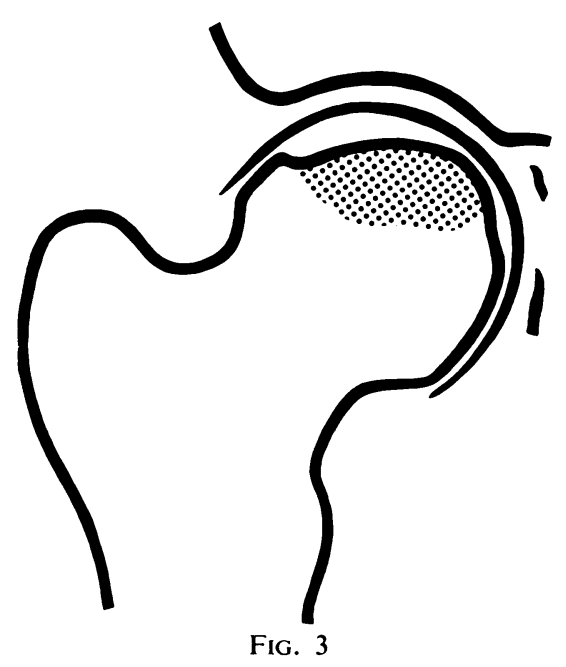

A diagram showing the principle of the "adjusted cup", the outer surface exactly congruent with the acetabular cartilage, the inner surface snugly fitting the sound part of the head. head and bearing on its sound portions (Fig. 3). The external diameter of the cup must be exactly equal to the interior diameter of the acetabulum. The head is fashioned with a reamer to make it equal the internal diameter of the cup or fractionally more. When the cup is hammered on and reduced it should move with the head in the acetabulum.

To achieve this, six sizes of cup are used, ranging from 40 to 50 millimetres with intervals of 2 millimetres. The hip is exposed by a lateral or a posterior approach, the head dislocated, the acetabulum measured exactly and the head reamed to the interior dimensions of the cup. The cup is hammered on to the head, on which it must be firmly secure. After reduction, the capsule is closed and the glutei are sutured as well as the fascia and skin. Light traction is generally applied for a week, but active flexion and rotation movements are started from the first days. The patient is allowed up on crutches after ten days and bears weight after one month.

\section{RESULTS}

First results were published in Declerck's Thesis (1972) and by Thomine (1973). Between 1964 and 1969 eighty hips in sixty-five patients have been treated by this operation and followed from one to six years. All these hips with idiopathic necrosis had some collapse of the head (grade II); 15 per cent were more advanced, with gross deformity of the head and commencing alteration of the acetabulum (grade III).

Pain was relieved in 75 per cent of the eighty hips, partially in 10 per cent, slightly or not at all in 15 per cent. Mobility was maintained in all cases where it was good before operation; when there had been some stiffness, it was improved in thirteen cases, unchanged in three and worse in two. Gait was dependent on pain. Altogether twenty-nine hips are completely painless, permitting normal mobility and gait, and twenty-four are practically painless, with some limitation and occasional limp. This means 66 per cent of complete or nearly complete success. Thirteen cases (16 per cent) are improved but still have some pain and limp. Another thirteen cases must be considered as failures. 
This relatively high percentage of failures may be related to the free indications for this operation, which has been used in practically all our last cases. To discover whether better selection could improve the results, the cause of failure has been investigated in all cases of necrosis of the head treated by "adjusted cup" arthroplasty. This means that the following considerations are based on ninety-nine such operations, eighty for idiopathic and nineteen for post-traumatic necrosis.

Radiographic changes after the operation may give some idea of the cause of poor or bad results. Rotation of the cup into a varus position was found in 57 per cent of the cases, and into valgus in 6 per cent. There was no definite correlation between this displacement and the functional result.
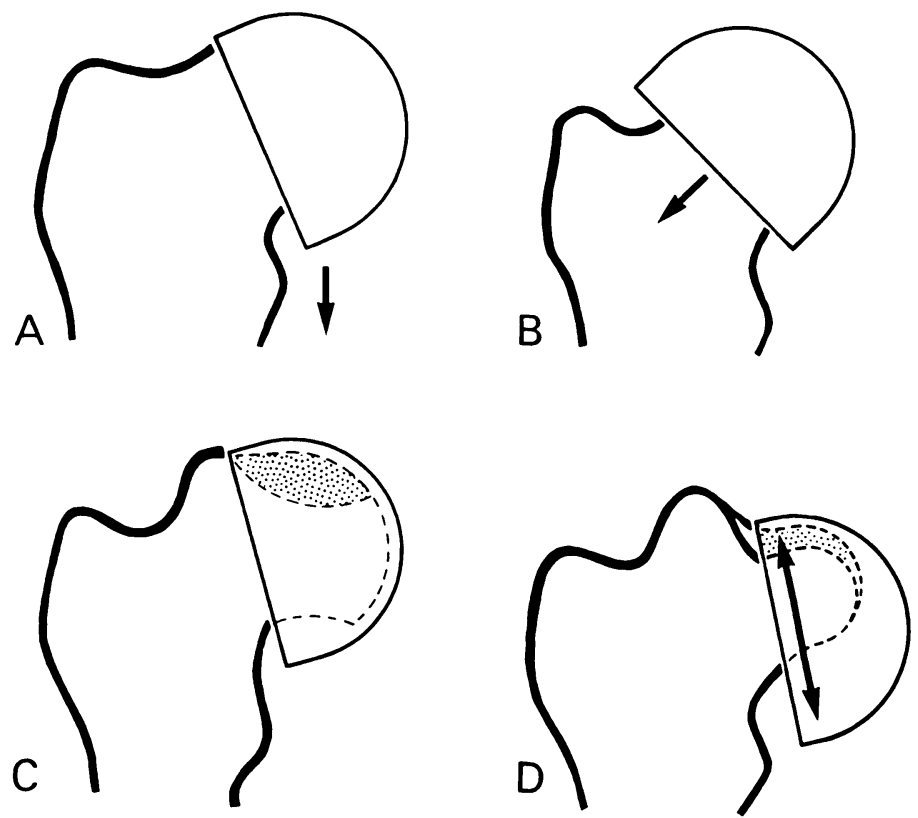

Fig. 4

A diagram of various radiographic changes that may be seen in the months or years following "adjusted cup" arthroplasty. A-vertical absorption of the head: B-axial absorption of the head; C-subluxation of the cup: and D-pathological fracture of the neck.

Absorption of the head under the cup appeared probable when the cup displaced laterally towards the greater trochanter from shortening of the neck (axial absorption) or toward the lesser trochanter (vertical absorption) (Fig. 4). The former was present in about half the cases, the latter in a third. When moderate, neither type of displacement influenced the functional result.

Subluxation of the cup occurred in nine cases; the necrotic femoral head came partly out of the cup, which sank down on the collapsed medial portion of the head. A pathological fracture in the medial portion of the head, under the depressed necrotic segment, occurred in three cases, probably because the avascular necrosis extended down to the inferior part of the head (Fig. 4).

These last twelve cases and two cases of pronounced absorption of the head are among the nineteen clinical failures of the ninety-nine "adjusted cup" arthroplasties. Four cases of absorption are among the fifteen poor results.

Alteration of the acetabulum may be another cause of an imperfect result. In twenty-five cases the cup did not fit the acetabulum exactly, being either too big (fifteen cases), with erosion of the acetabular roof as a rule, or too small, with some medial displacement of the cup. This imperfection was found in none of the failures but in six cases with an imperfect functional result. 
In twelve cases the acetabulum was not perfectly sound at the time of the operation. Consecutive radiographs showed improvement of the joint space in seven cases, while the arthrosis advanced in five cases. Nevertheless two cases had a good functional result and three were fair.

Periarticular ossification was found in two cases with poor functional results. Finally, in two cases of poor results no apparent reason could be found.

From this review it appears that further evolution of the necrosis and collapse of the head under the "adjusted cup" is the main cause of failure of the operation. It may be that the femoral head does not offer the cup sufficient support due to the extent of the necrosis. If so, it would be valuable to estimate from the pre-operative radiographs the amount of sound surface of the head, especially over its lateral aspect, with the object of selecting favourable cases. This appreciation was tried by measuring the arc of apparently sound surface lateral to the necrosis, the "bearing sector", in fifteen of the seventeen cases of failure. It was 20 degrees or more in only three cases, while in twelve cases it was less than 20 degrees and in nine cases less than 5 degrees.

If in the whole series of ninety-nine operations one takes only those cases where the sound "bearing sector" was 20 degrees or more, and the right size of cup was used, 88 per cent of the results are good, 7 per cent fair and 5 per cent poor. If on the other hand the preoperative findings are unfavourable and confirmed by direct inspection at arthrotomy, then it would seem best to proceed with prosthetic replacement, preferably total, even in a comparatively young patient.

\section{SUMMARY}

1. The surgical treatment of idiopathic aseptic necrosis of the femoral head has been reviewed in the light of experience gained from 240 hips operated upon.

2. When pain is not severe and the necrosis of bone as seen in serial radiographs is not rapidly progressive, simple observation and palliative medical treatment are indicated.

3. When pain disables the patient and collapse of the head is progressive, operation is indicated. If radiographs show necrosis limited to the anterior part of the head and sparing an arc of at least 20 degrees of the lateral part, either an osteotomy bringing the shaft into adduction and flexion or an "adjusted cup" arthroplasty is indicated, with a preference for the latter because it gives better results in a shorter time.

4. If at arthrotomy the necrosis appears to involve the posterior slope of the head, prosthetic replacement, preferably total, should be undertaken.

\section{REFERENCES}

AUbRiot, J.-H. (1973): Traitement par prothèses fémorales métalliques des ostéonécroses aseptiques primitives fémorales. Revue de Chirurgie Orthopédique et Réparatrice de l'Appareil Moteur (Paris), 59, Supplement 1, 81.

DeCLerCK, B. (1972): Traitement de l'ostéonécrose de la tête fémorale par la cupule ajustée. Thèse, Paris.

FICAT, P., and ARLET, J. (1972): Coxopathies ischémiques. Revue de Chirurgie Orthopédique et Réparatrice de l'Appareil Moteur (Paris), 58, 543.

Kerboull, M. (1973): L'ostéotomie intertrochantérienne dans le traitement de la nécrose idiopathique de la tête fémorale. Revue de Chirurgie Orthopédique et Réparatrice de l'Appareil Moteur (Paris), 59, Supplement $1,52$.

Merle D'Aubigné, R., and Frain, P. G. (1972): Théorie des ostéotomies. Revue de Chirurgie Orthopédique et Réparatrice de l'Appareil Moteur (Paris), 58, 159.

Merle d’Aubigné, R., Mazabraud, A., and Cahen, C. (1963): La nécrose idiopathique de la tête fémorale. Étude anatomo-pathologique et orientation thérapeutique. Semaine des Hopitaux de Paris, 39, 2773.

Merle d’Aubigné, R., Postel, M., Mazabraud, A., Massias, P., and Gueguen, J. (1965): Idiopathic necrosis of the femoral head in adults. Journal of Bone and Joint Surgery, 47-B, 612.

Rydell, N. W. (1966): Forces acting on the femoral head prosthesis. Department of Orthopaedic Surgery, Göteborg, Sweden.

Thomine, J. M. (1973): L'arthroplastie par cupule ajustée pour nécrose de la tête fémorale. Revue de Chirurgie Orthopédique et Réparatrice de l'Appareil Moteur (Paris), 59, Supplement 1, 61. 